\title{
A perspective on process synthesis: Challenges and prospects
}

\author{
Selen Cremaschi \\ Department of Chemical Engineering, The University of Tulsa, 800 South Tucker Drive, Tulsa, \\ Oklahoma 74104, USA
}

\begin{abstract}
This paper gives the author's perspective on some of the open questions and opportunities in process synthesis focusing on separation systems as the application. Driven by energy and environmental concerns and challenged by introduction of new raw materials, this author anticipates significant advances in: (1) novel approaches that integrate experimental studies and process synthesis activities, and multi-scale and surrogate models for accurately capturing the behavior of these unconventional mixtures, (2) systematic generation of alternatives for processing these mixtures, and (3) global, robust, and stochastic optimization for identifying the optimum alternative. This paper is an extended version of a conference paper (Cremaschi, 2014) presented at the $8^{\text {th }}$ International Conference on Foundations of Computer-Aided Process Design.
\end{abstract}

Keywords: Process synthesis, distillation sequences, separation systems, surrogate models

*Corresponding author: selen-cremaschi@utulsa.edu

\section{Introduction}

Since the introduction of the term "process synthesis" in the late 1960s, the process systems engineering (PSE) community made significant progress in the area. As depicted in Fig. 1, for given desired products and available raw materials, the process synthesis methods try to determine 
the combination of processes to obtain the products starting from the raw materials while satisfying a predetermined objective, which is in most cases minimum cost, but may also be minimum energy consumption or maximum yield among others. One of author's favorite quotes by Westerberg (2004) about process synthesis reads as follows: "Process synthesis is very much the fun part of engineering. It is where one invents the structure and operating levels for a new chemical manufacturing process."

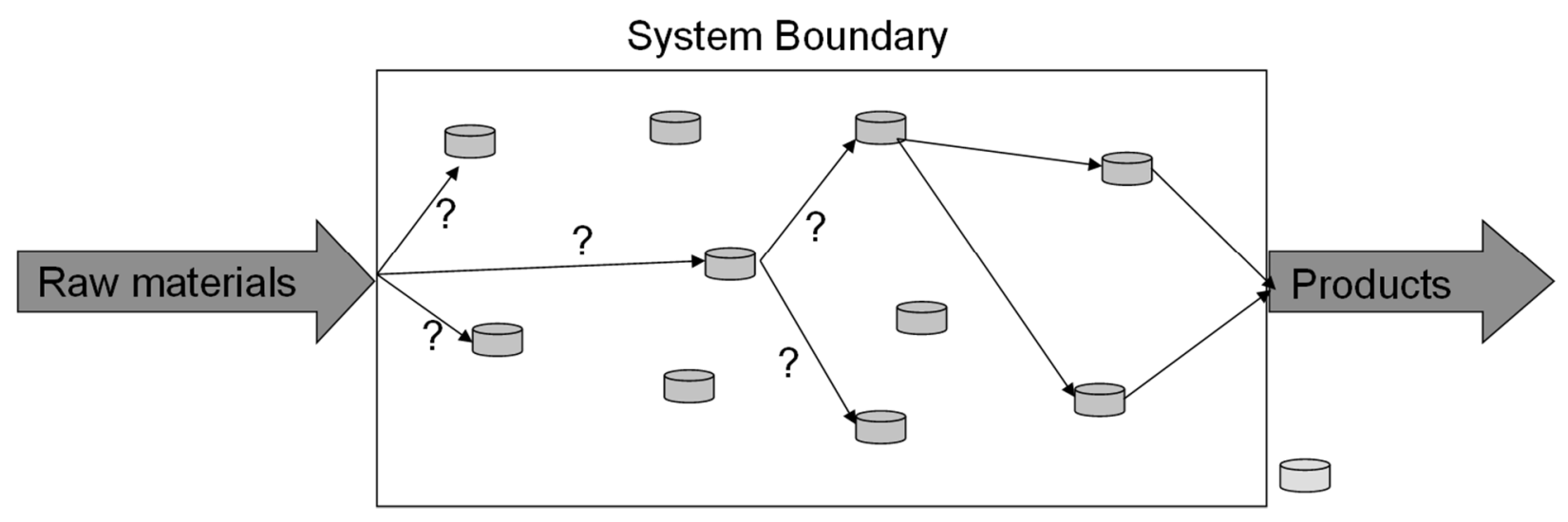

Figure 1. A graphical representation of process synthesis

The first review in process synthesis by Hendry et al. (1973) had over 60 articles. The $12^{\text {th }}$ Annual Symposium on Computer Applications in Chemical Engineering had 10 contributed papers in process synthesis area - three dealing with heat exchanger networks, two in synthesis of separator networks, four in overall flowsheet synthesis, and one industrial experience paper that discussed the application of the existing synthesis approaches to heat exchanger, separator, and reactor networks - out of 115 overall contributions (Motard, 1979). A recent search in the abstract and citation database Scopus yielded well over 2000 publications from 1972 to 2013. Fig. 2 shows the annual number of publications in the four main areas that our community focused on over the last 
four decades: reactor network, distillation train, heat-exchanger network, and overall flowsheet synthesis. Fig. 2 suggests at least a linear increase in the total number of publications in the process synthesis area over the last two decades, a little over half of the contributions in heat-exchanger network synthesis, followed by contributions in separator sequence and overall flowsheet synthesis.

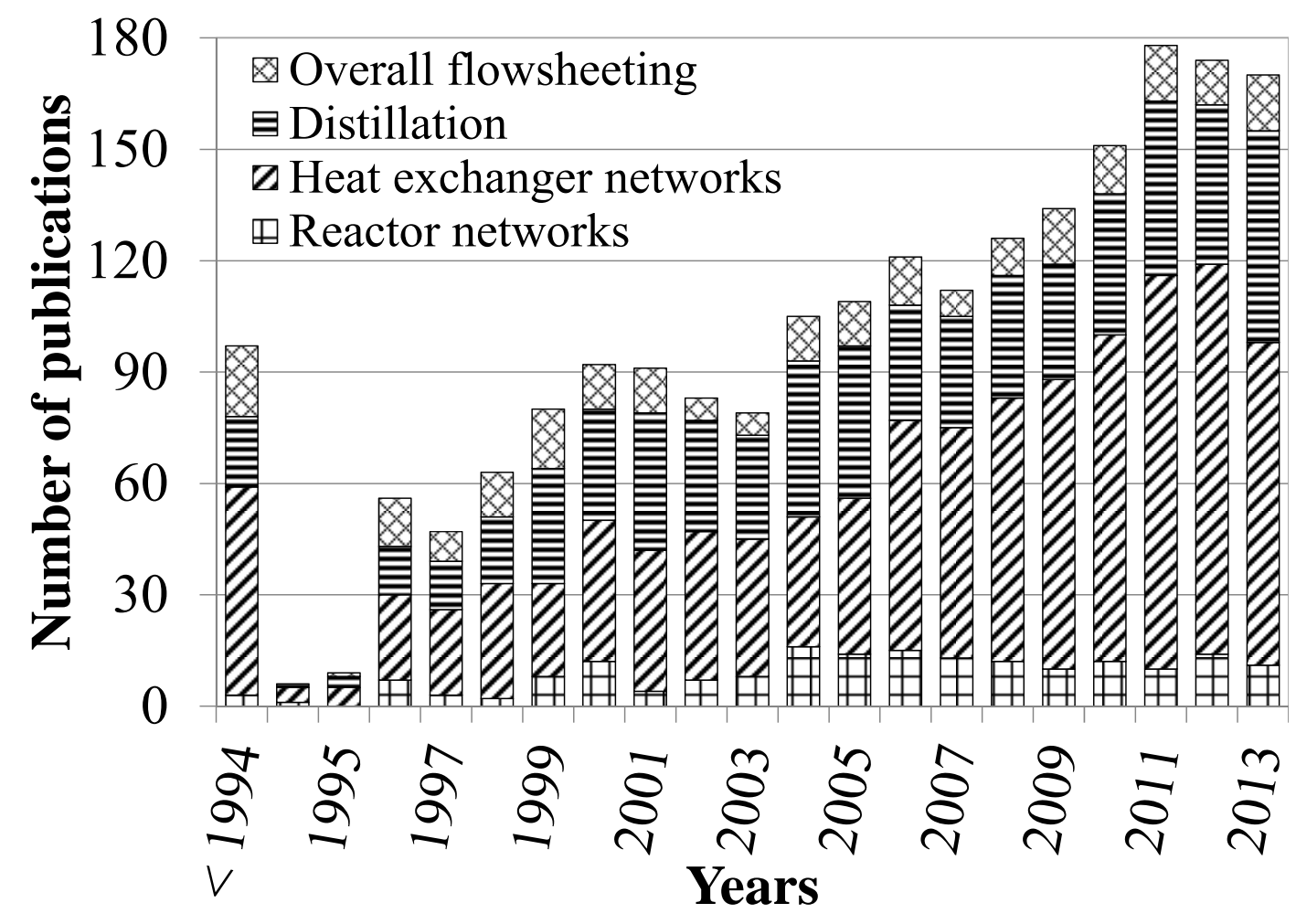

Figure 2. Number of process synthesis publications (1972 - 2013) - Reactor networks: search in All Fields for the exact phrase "reactor network synthesis", distillation: search in All Fields for either of the following exact phrases "distillation column synthesis", "separation network synthesis", "distillation sequence synthesis", "distillation synthesis", "separation train synthesis", heat-exchanger networks: search in All Fields for the exact phrase "heat exchanger network synthesis", and overall flowsheeting: search in All Fields for either of the following exact phrases "flowsheet synthesis", "complete flowsheet synthesis", "general flowsheet synthesis". 
Clearly, a review of the existing literature in this paper is neither feasible nor the intent. However, the author refers the interested reader to a selected subset of excellent reviews and perspective papers on: reactor and reactor-separator network synthesis focusing on attainable region construction (Feinberg, 2002), synthesis of heat integration networks (Furman \& Sahinidis, 2004; Morar \& Agachi, 2010), water network synthesis (Jeżowski, 2010), distillation-based separation sequence synthesis (Skiborowski et al., 2013), and general process synthesis approaches focusing on their substantial potential for synthesis of sustainable and environmentally-friendly processes for energy and chemicals production (Grossmann \& Guillén-Gosálbez, 2010; Z. Yuan \& Chen, 2012; Zhihong Yuan et al., 2013).

The goal of this paper is to provide the author's perspective on some of the open issues as they relate to process synthesis using separation-system synthesis as an example domain. It is this author's opinion that process synthesis holds unique potential to contribute to the solution of some of the "Grand Challenges for Engineers" identified by National Academy of Engineering, and separation systems will play a vital role for addressing energy, water, and pharmaceutical needs, and carbon capture problems. A brief overview of process synthesis approaches is given in next section. Section three discusses open issues and opportunities in separation-systems synthesis. The last section offers concluding remarks.

\section{2. Process synthesis approaches}

The approaches used to address process synthesis problems can be categorized into one of the two broad groups: (1) hierarchical decomposition based heuristic approaches, and (2) mathematical programming based approaches. The early contributions to the process synthesis area were mostly 
development and implementation of systematic hierarchical decomposition approaches to remove the chemical and physical differences between the raw materials and the products, e.g., meansends analysis (Siirola \& Rudd, 1971), and the 5-level decision hierarchy to conceptual design (Douglas, 1988). The economic short-cut evaluations of the alternatives were carried out at each decision level to reduce the number of alternative flowsheets. Although powerful and generally yielding near-optimal processes, the heuristic based decomposition approaches do not consider the interactions between different levels, and cannot guarantee that the end design is the best possible for the selected performance metric, mostly an economical one.

Tied mostly to increasing computing power and advances in formal optimization techniques, mathematical programming approaches, i.e., superstructure optimization, were developed to incorporate the interactions between different design levels and their overall effect on the selected performance metric. In superstructure optimization, the overall process network is determined in one simultaneous mathematical programming problem by optimizing a desired performance metric given the initial superstructure of the system, the material flow through each interconnection, operating conditions, and other design parameters for each equipment (Barnicki \& Siirola, 2004).

The first step in superstructure optimization is to construct a map of all the design alternatives using an appropriate representation. The commonly used representations for process synthesis problems are State-Task, State-Equipment, and Resource-Task Networks, and Generalized Modular Framework. More recently, Unit-Operation-Port-Stock Superstructure and groupcontribution based representations are proposed. A brief review of these representations can be 
found in Fahmi et al. (2014). Once the alternatives are mapped, the next step is to translate this representation to a mathematical programming formulation, mostly resulting in a large-scale nonconvex mixed integer (non)linear programming (MINLP) problem, whose objective is generally an economic one.

The resulting optimization formulation, in most cases very difficult to solve, fostered the development of a myriad of tailored and general optimization algorithms, e.g., the outerapproximation approach for obtaining local solutions (Viswanathan \& Grossmann, 1990), and BARON - Branch-And-Reduce Optimization Navigator - (Tawarmalani \& Sahinidis, 2005), or ANTIGONE - Algorithms for coNTinuous/Integer Global Optimization of Nonlinear Equations - (Misener \& Floudas, 2014) for obtaining global solutions. Although superstructure optimization is theoretically a very powerful approach, its penetration to the industrial applications has been limited due to two major drawbacks: (1) the optimum can only be obtained if the superstructure contains the optimum when constructed (Barnicki \& Siirola, 2004), (2) the difficulty of solving the resulting MINLPs (Henao \& Maravelias, 2010). Both decomposition-based and mathematical programming approaches have their own limitations, and their strengths may be complementary. Some of the recent contributions focus on combining these approaches for synergistic effects (Zhihong Yuan et al., 2013).

\section{Challenges and opportunities - Separation-system synthesis}

Top chemical companies are shifting their focus from bulk chemicals to specialty products. For example, BASF expects to increase the share of "customized products, and functionalized materials and solutions" sales to $70 \%$ of its total by year 2020 . They continue to acquire specialized 
"close-to-end-user" businesses as they exit from some of the commodity markets (e.g., fertilizers) (BASF, 2014). In a similar trend, the Dow Chemical Company is "carving-out" its chlorine and epoxy assets reducing its commodity chemicals foot-print while it continues to grow in downstream specialty products in integrated plastics, electronics, and agriculture (Company, 2014). Bayer recently announced that they will become a "pure Life Science company", focusing their business entirely on products that enhance human health and nutrition (Bayer, 2014). Dupont continues to focus its product offerings on agriculture and nutrition developed using the latest advancements in biotechnology (DuPont, 2013). These trends suggest that the raw material and product portfolios of chemical process industry may grow and be quite different compared to today's in the near future.

The world population is expected to grow to 9 million by 2050 causing considerable increases in energy and natural resource demands. Based on the report titled Environmental Outlook to 2050 by The Organisation for Economic Co-operation and Development (OECD), the world's economy is expected to grow four times its current size using $80 \%$ more energy by 2050 . The climate change due to increased greenhouse gas emissions from economic growth, loss of biodiversity, water scarcity, and substantial increases in $\mathrm{SO}_{\mathrm{x}}$ and $\mathrm{NO}_{\mathrm{x}}$ emissions in developing economies are identified as the four main environmental challenges. Although some of these environmental challenges require policy changes and cannot be addressed with technological advances, some provide unique opportunities for the process systems engineering community, especially in process synthesis area. The reduction of greenhouse gas emissions, and increasing energy and water usage efficiency of our processes via innovative process synthesis and design are some example areas. 
In this section, the author's perspective on some of challenges and the corresponding opportunities for process synthesis resulting from the raw material and product diversification, and energy and environmental concerns are discussed using synthesis of separation systems as the specific domain. The selection of separation-systems process synthesis is motivated by the high capital costs and energy consumptions of these systems (Agrawal, 2001). Most discussion in this section may be extended to process synthesis of other systems.

\subsection{Distillation sequences}

Distillation, whose roots are believed to be from purification of ethanol for alcoholic beverage production in $\sim 800 \mathrm{BC}$ (Kiss, 2014), is used to perform majority of the separations in chemical process industry. Currently, it is estimated that $90 \%$ of all separations are carried out by distillation unit(s) (Felbab et al., 2013). Figure 3 provides possible basic distillation trains for separation of a tertiary mixture. The synthesis of distillation units and trains has received considerable attention from the PSE community. Early contributions focused on identifying distillation sequences for ideal multicomponent mixtures focusing on short-cut design models, while the majority of recent research work uses superstructure optimization with rigorous distillation models (Skiborowski et al., 2013). The resulting large scale nonconvex MINLPs have motivated the investigation of effective problem formulations and efficient solution approaches (Grossmann et al., 2005). A brief review of the PSE contributions to the synthesis of distillation column sequences up to 2004 is summarized by (Westerberg, 2004). 


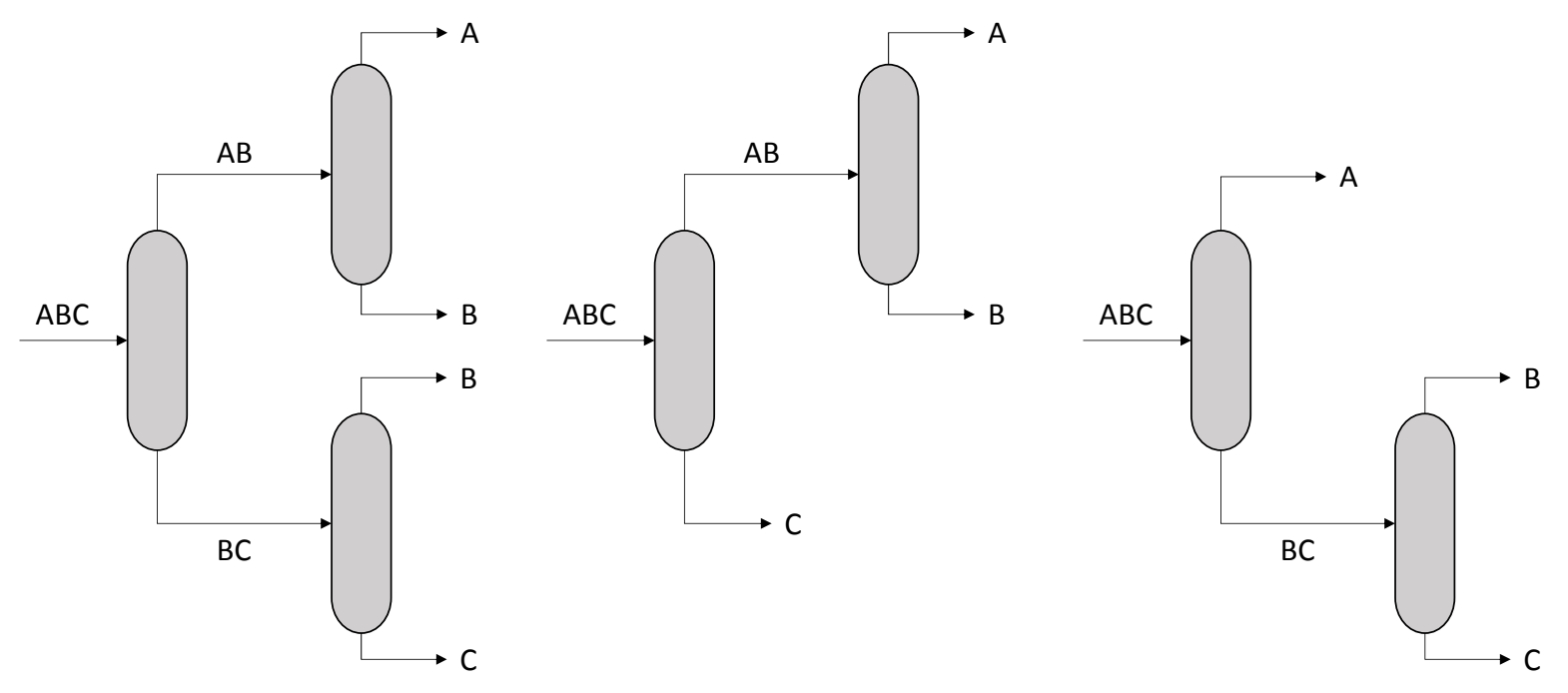

Figure 3. Basic distillation column configurations for separation of a tertiary mixture.

Distillation is a highly energy intensive and inefficient process as it requires addition of high quality energy at the reboiler and rejects low quality energy at its condenser. It also has a high capital cost due to large equipment used (Felbab et al., 2013). These shortcomings fuelled the identification of complex and unconventional distillation configurations. Kiss (2014) recently summarized some of the suggested and implemented (in industry) configurations under the following seven groups: (1) heat pump assisted distillation, (2) heat integrated distillation, (3) membrane distillation, (4) HiGee distillation, (5) cyclic distillation, (6) dividing wall columns, and (7) reactive distillation. Figure 4 shows some example configurations. The process synthesis efforts mostly focused on identification of thermodynamically superior (compared to conventional columns) and/or optimum heat pump assisted (e.g., (Felbab et al., 2013)), heat integrated, dividingwall (e.g., (Caballero \& Grossmann, 2013; Shenvi et al., 2013)), and, though fewer contributions, reactive columns (e.g., (Urselmann et al., 2011)). As chemical process industry continues to increase the energy efficiency of its separations, reduce its greenhouse gas emissions and water usage in the future, there are tremendous opportunities for advancing conventional and unconventional distillation train synthesis. As the heat and mass integration of the separation trains 
increase reducing available degrees of freedom for control, the controllability considerations during the process synthesis step will become essential. Therefore, the size and the character of the process synthesis problem may change demanding new representation and solution approaches.

The process synthesis of distillation or in general separation trains can be separated into two major tasks: (1) generation of sequence alternatives that contain the optimum, and (2) the identification of the optimum from the alternatives. It is evident that achieving task one successfully is a necessary condition for optimum separation-system synthesis. There have been significant contributions for accomplishing task one for multicomponent zeotropic mixtures generally focusing on conventional or thermally integrated columns. For example, in a recent publication, Caballero and Grossmann (2013) developed a set of logic constraints using state-task network to generate all basic, thermally integrated, and single dividing-wall column configurations for separating an $M$ component zeotropic mixture to $N$ components $(N \leq M)$. They used these constraints in a generalized disjunctive model (GDP) to find the optimum separation train. The objective was to minimize total annualized cost considering the effects of condenser and reboilers. Agrawal and coworkers introduced a supernetwork model to generate all basic column configurations (Giridhar \& Agrawal, 2010) followed by a matrix method for generating both basic and thermally coupled distillation configurations (Shah \& Agrawal, 2010). The extension of these systematic approaches to alternative generation for nonideal mixtures has not been extensively studied, and is an open challenge. Once the alternatives are generated, the common approach to achieve the second task, identification of the optimum from the set of alternatives, is solving a mathematical programming problem with a selected objective, e.g., minimum cost or energy. The 
resulting formulation is generally a large scale, highly nonconvex MINLP, which requires significant computational resources to solve to global optimality (Caballero \& Grossmann, 2013). Hence, it is expected that process synthesis of separation systems will contribute to the continued progress in global solvers.

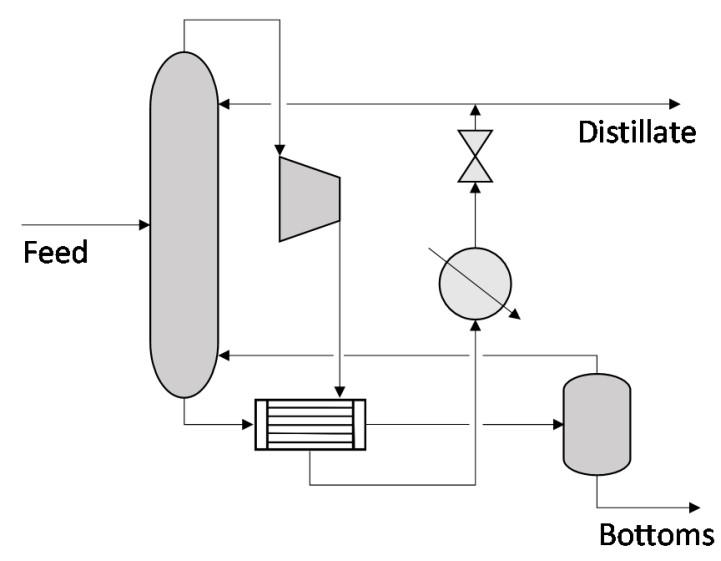

(a)

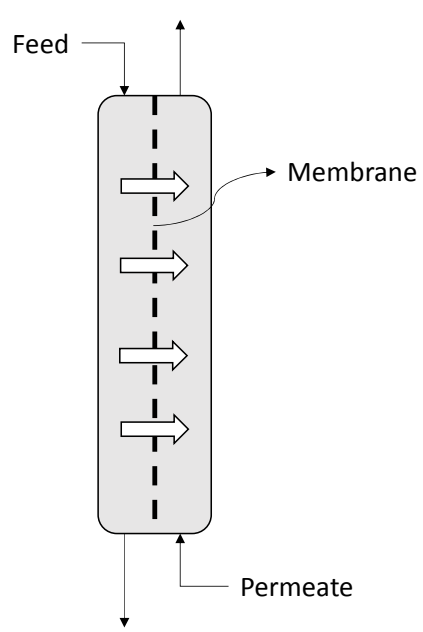

(c)

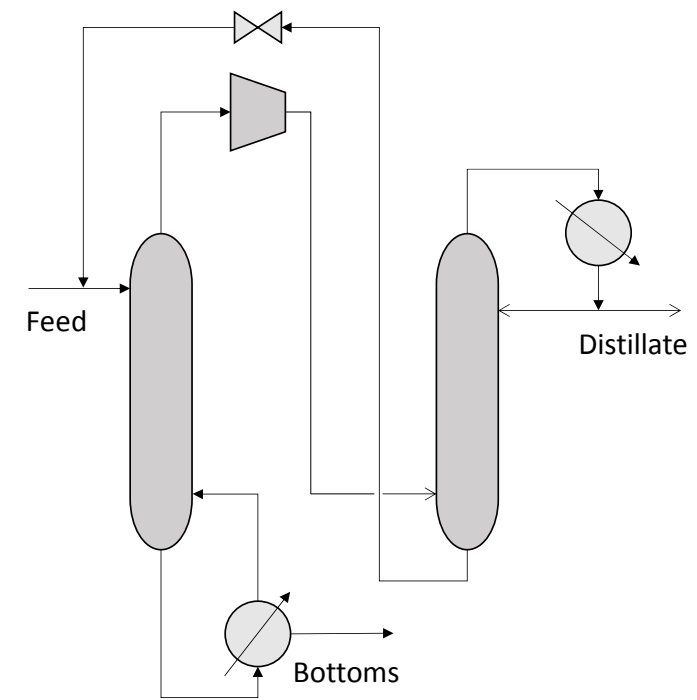

(b)

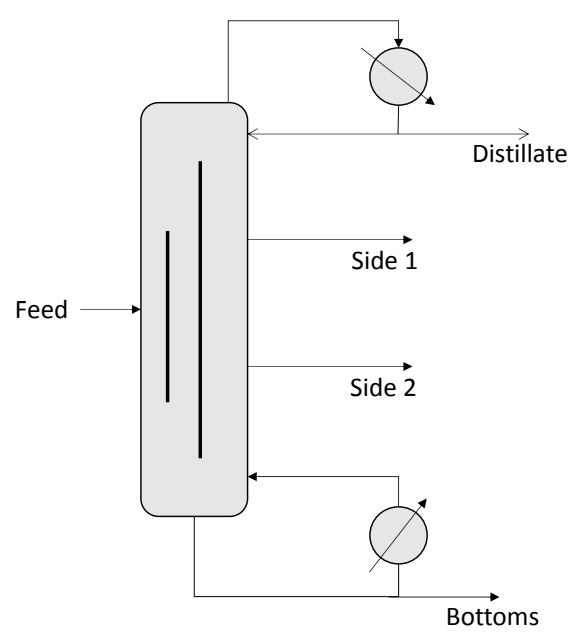

(d)

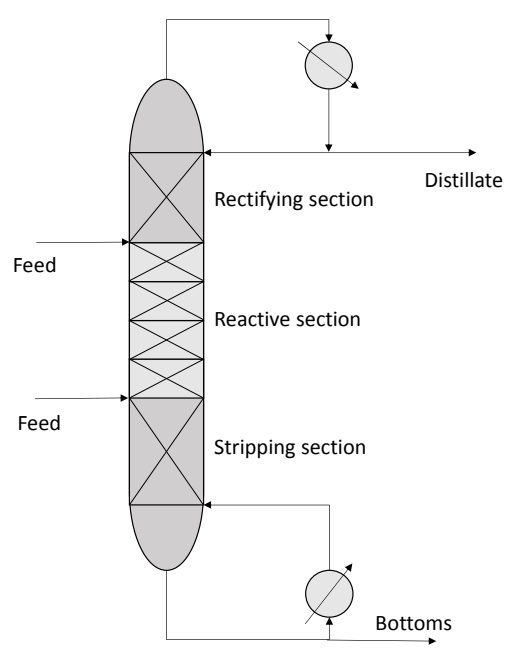

(e)

Figure 4. Example unconventional distillation columns, (a) vapor recompression distillation, (b) heat integrated distillation, (c) membrane distillation, (d) dividing wall columns, and (e) reactive distillation 


\subsection{Hybrid separation sequences}

Although distillation sequences govern most industrial separations, there are many other separation processes that are used such as liquid-liquid extraction, adsorption, and membranes. The process synthesis of these separation processes, either as homogenous trains or as hybrid sequences, has received limited attention to date. An excellent review by Skiborowski et al. (2013) discusses in detail the status quo of process synthesis tools available along with the open challenges for the process synthesis and design of distillation-based hybrid separations. The authors defined these separations as the ones that include distillation and other unit operations (See Figure 5 for example configurations). Specifically, they discuss combinations of distillation with liquid-liquid extraction, decantation, crystallization, and membranes. The authors highlight the scarcity of systematic approaches available to generate the alternative sequences, short-cut models to rapidly screen the alternatives, and optimization-based approaches to identify the best process using rigorous steady-state and/or rate-base models in all of these systems. As summarized by the authors, most contributions in synthesis and design of these hybrid processes rely on graphical analysis of the mixture thermodynamics, and hence, are limited to at most four component separations. As chemical process industry incorporates new feedstocks such as biomass or the separation of natural products from specifically engineered organisms, the number of components in these mixtures is expected to increase limiting the use of these graphical approaches. Furthermore, the thermodynamic data or models to adequately model the behavior of some of these mixtures may not be readily available. 


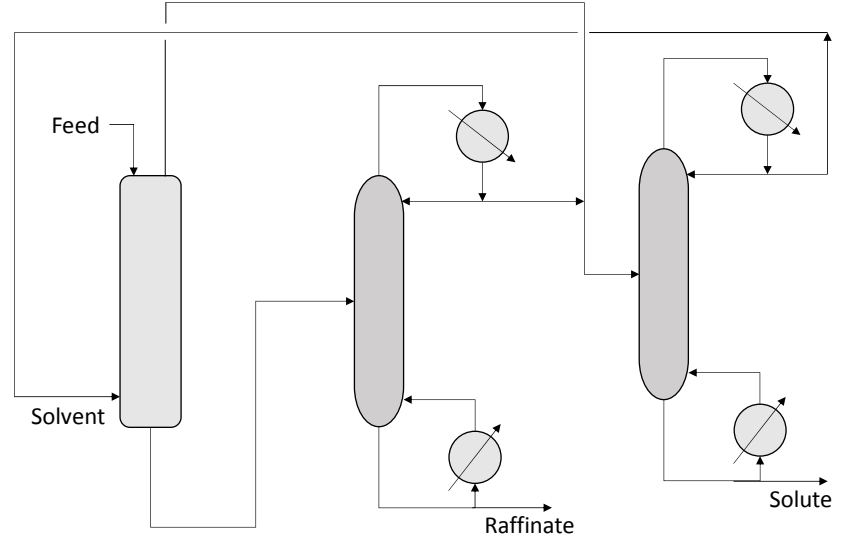

(a)

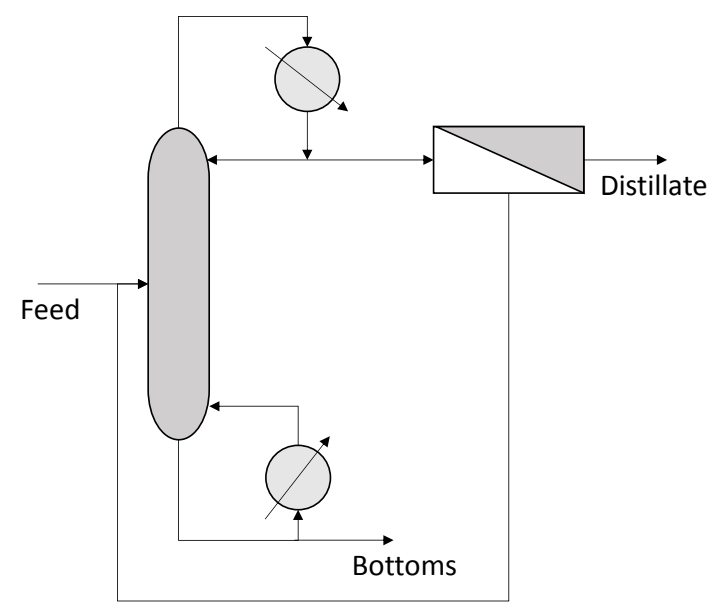

(b)

Figure 5. Example hybrid separation sequences (a) liquid-liquid extraction and distillation, and (b) membrane-assisted distillation

Some unit operations include additional design degrees of freedom compared to distillation, e.g., the selection of the optimal solvent or membrane. There have been considerable advances in computer-aided molecular design (CAMD) to address the solvent synthesis problem. However, the solvent selection affects the separation system design, and the separation system design affects the optimum solvent selection. A sequential approach may not yield the optimum separation system. Similar arguments can be made for systems including membrane separations. Although there have been advances for combining product and process design especially considering solvent design for extraction, e.g., Bommareddy et al. (2010), the contributions for other hybrid-separation sequences have been limited. The problems with the additional degrees of freedom become very big quickly, and our ability to solve these problems is limited. In this author's opinion, the complexity introduced by all these factors will necessitate the use and development of novel multiscale approaches to accurately capture the behavior of these processes, and amplify the importance 
of surrogate models and derivative-free optimization approaches for their synthesis (Biegler et al., 2014).

\subsubsection{Surrogate Models for Process Design}

Using a broad definition, surrogate models represent functional relationships between input and output variables. They are generated by statistical combination of the input values and the corresponding output values that were obtained either experimentally or by solving rigorous firstprinciple models that define the systems (Qian et al., 2006). There has been a renewed interest in using surrogate models for process synthesis, design and optimization within the last decade. For example, Fernandes (2006) used neural networks to model the Fischer-Tropsch process. The relationship between the amount of gasoline or diesel production and pressure, hydrogen to carbon dioxide ratio, and the space velocity, which are traditionally modeled using complex kinetic mechanisms, is represented by a neural network and the surrogate neural network model is used to maximize the production amount. Henao and Maravelias (2011) represented each and every unit operation with neural networks as the surrogate models, and demonstrated the feasibility of using these surrogate models with several examples. The author's group also worked in process synthesis and design using surrogate models. We analyzed the impact of different amine absorbents and their concentrations, the absorber and stripper column heights and the operating conditions on the cost of $\mathrm{CO}_{2}$ recovery plant for post-combustion $\mathrm{CO}_{2}$ removal combining second-order response surface surrogate models and steepest descent routine iteratively (A. Nuchitprasittichai \& Cremaschi, 2011), and using artificial neural networks - ANNs (Aroonsri Nuchitprasittichai \& Cremaschi, 2013), synthesized a biodiesel production process using ANNs (Fahmi \& Cremaschi, 2012), and designed a packed-bed column photobioreactor for algae growth (Smith et al., 2012). 
Most work in this area to date uses the traditional approach for surrogate model generation: (1) Collect input/output data based on a selected experimental design, (2) Select surrogate model form and determine its parameters, (3) Validate the surrogate model performance using an independent data set, and (4) Use the surrogate model if successful; discard all data and re-start the whole process from the beginning if not successful. However, this one-shot approach becomes expensive as the complexity of the original model/system increases and as the cost of rigorous model evaluation/experiments increases. Sequential surrogate model construction approaches start with a small number of input/output pairs. They construct an initial surrogate model, and increase the number of input/output pairs iteratively constructing and testing the performance of the surrogate model at each iteration. By systematically increasing the sample size while maintaining previous sampled points, sequential methods have been shown to require fewer observations, i.e., function evaluations, than one-shot procedures, and hence, reducing the total time required for data collection.

In sequential surrogate model construction, there are two main questions: (1) what is the minimum number of samples that are required to build an acceptable surrogate, and (2) where should these samples be located? The answer to both of these questions greatly impact the overall cost of surrogate model construction. Two approaches can be considered when collecting the input/output pairs, the exploration and the exploitation of the search space. The former enables the proper coverage of the search space, and hence, aids in the identification of nonlinear/discontinuous, i.e., interesting behaviors of the underlying function/phenomena, whereas the latter becomes very important for correctly quantifying the magnitude of these interesting behaviors. Another 
important criteria when considering sample collection for surrogate model construction is how the approach scales with high dimensions. This criteria becomes especially important for process synthesis and design applications as these problems generally have high number of input/output dimensions. A recent contribution by Eason and Cremaschi (2014) discusses potential impacts of exploration and exploitation in surrogate-model construction, and introduces two algorithms that adaptively locate new input/output pairs for surrogate model construction and scale well to high dimensions.

There are significant open challenges before we can routinely use surrogate models for solving process synthesis problems. The author envisions that the questions related to the balance of exploration and exploitation as well as the type of exploitation will be addressed in the near future, and the identification of the appropriate surrogate model form and its parameters may be a midterm goal in this area. The two big challenges in this field, namely the identification of appropriate termination criteria for stopping sequential surrogate model training, and proving that the solution obtained using the surrogate model is the global/local optimum, are interrelated, and, in this author's opinion, can be viewed as long-term goals that require considerable research and theory development in this field.

\subsection{Separation of unconventional mixtures}

The synthesis of separation systems to extract natural products from plant raw materials or microorganisms has received very little attention to date. Two recent publications in this area focused on synthesis of protein purification processes. Polykarpou et al. (2012) developed an MILP model to determine the minimum number of chromatographic steps for separating a mixture 
of proteins with known physicochemical properties. They also used a weighted objective that considers the number of steps along with the final purity and recovery of the products. One of the main limitations of this approach is the assumption that the physicochemical properties of each component in the mixture is available, which is rarely the case. Nfor et al. (2013) proposed a hierarchical decomposition approach that combines experimental studies with mathematical programming approaches to synthesize separation sequences for protein purification. They divide the problem into five levels: (1) purification task definition, (2) resin selection and model parameter determination, (3) generation of the alternative separation sequences and elimination of least promising alternatives, (4) development of optimization model for determining the optimum design for each alternative, and (5) comparison of the optimum designs of the alternatives to determine the best one. Malwade et al. (2012) introduced a heuristic-based approach to synthesize a separation train for recovery of natural products from plant biomass. The authors emphasized the importance of integration of process analytical technology for physicochemical data collection with knowledge based heuristics for the recovery process synthesis.

With advances in directed evolution techniques (Johannes \& Zhao, 2006) and the growth of the biopharmaceutical sector (Nfor et al., 2008), the process synthesis of recovery and purification of the bioproducts and proteins that are produced by cell cultures is becoming an important problem to manage their overall production cost. These mixtures are considerably different compared to the downstream feeds of traditional chemical process industry: (1) the desired product concentration is generally very low and there may be a host of compounds with very similar physical and chemical characteristics to the desired product, (2) the mixtures may contain hundreds of compounds with little or no thermodynamic or kinetic data, and (3) the desired products and the 
other compounds in the mixture are easily degradable (Malwade et al., 2012). Hence, the separation trains designed to recover the products from these mixtures include processes that are not commonly used in traditional chemical industries, and the process synthesis of these systems may require development of new approaches. Given the scarce data on these processes and the mixture properties we are trying to separate, the author suspects that a stronger integration of experimental studies with the process synthesis activities is expected to address some of these challenges. Here, the sequential refining of the model with adaptive experimental designs may play a significant role. The premise of this approach was demonstrated for optimization of nanoparticle deposition process by Casciato et al. (2012). The process synthesis activities of these processes will certainly experience higher levels of data uncertainty demanding use and development of optimization approaches under uncertainty, such as robust optimization, and stochastic and multi-parametric programming (Domínguez \& Pistikopoulos, 2013). Furthermore, given data uncertainty, further research in the uncertainty quantification and propagation areas is expected.

The adaptive experimental designs developed with feedback loop from the process synthesis step to the experimental stage may result in the gradual resolution of uncertainty. The incorporation of this decision-dependent uncertainty in process synthesis yields large-scale stochastic problems under endogenous and exogenous uncertainties, for which efficient solution algorithms are not available. Most contributions in optimization under endogenous uncertainty to date focus on problems where only the resolution of uncertainty is decision-dependent (e.g., (Christian \& Cremaschi, 2014; Colvin \& Maravelias, 2008; Gupta \& Grossmann, 2014)). However, the problems resulting from the integration of experimental studies with process synthesis activities 
through a feedback loop results in an optimization problem with endogenous uncertainty, where the distribution parameters and the observation of uncertainty depends on the decisions. The literature that deals with this class of problems, where the decisions impact the distribution parameters of the uncertainty, is scarce. Viswanath et al. (2004) developed a two-stage stochastic programming formulation to minimize the expected shortest distance between start and end nodes of a network under random failures. The survival probability of a link in the network could be increased by investing on it at the first stage. They approximated the objective using the first order terms of the multilinear function obtained by relaxing the integrality constraints of the investment decision variables and using Taylor series expansion, and used an iterative solution algorithm to improve the approximate objective function value. Held and Woodruff (2005) introduced the problem of multi-stage interdiction of networks, where the structure of the network is uncertain. The objective was to maximize the probability of interdicting the flow of information or goods through the network, and probability distribution of the next stage network structure depends on the interdiction decisions. The authors investigated the use of heuristic approaches. The author expects a growth in this field partially motivated by the process synthesis problems resulting from these specific integrations.

The integration of experimental studies with process synthesis activities may also foster the use of surrogate models at the early stages of the experimental studies, and may provide assistance in constructing experimental test matrices for maximizing information gain from the experiments. In turn, the resulting surrogate models may eliminate big chunks of search space from consideration for process synthesis as more experimental data become available. Therefore, similar arguments 
about the open challenges regarding surrogate model construction (Section 3.2.1) become valid for process synthesis of separation of unconventional mixtures.

The author also expects that the strong integration of experimental studies with the process synthesis activities will results in more research in development and use of novel data clustering approaches for identification of relevant data for model discrimination and validation, innovative expansion and exploitation of model evaluation techniques to identify correct models for the data set at hand, and identification and expansion of uncertainty representation theories and propagation techniques to properly quantify the confidence intervals of model outputs.

\subsection{Process intensification}

Process intensification is considered to be one of the most promising progress paths for development of more sustainable chemical processes (Van Gerven \& Stankiewicz, 2009). There are different definitions of process intensification (PI) in the literature, one this author favors is "Any chemical engineering development that leads to a substantially smaller, cleaner, safer, and more energy efficient technology." (Stankiewicz \& Moulijn, 2000). Excellent overviews of PI can be found elsewhere (Lutze et al., 2010; Van Gerven \& Stankiewicz, 2009).

Two of the three industrially implemented PI technologies include separations, reactive distillation and dividing-wall columns (J. Harmsen, 2010). In fact, the top spot for process intensification belongs to reactive distillation, more specifically to the Eastman process that replaced an overall methyl acetate production process with a single distillation column yielding $80 \%$ reduction in total capital and energy costs (G. J. Harmsen, 2007; Kiss, 2014). There have been significant 
contributions to achieve systems with PI (see (Lutze et al., 2013) for a brief review). The development of systematic approaches for synthesis of systems that will achieve PI seems like an ill-posed goal as PI intrinsically entails out-of-the-box thinking. However, recent advances in phenomena- (Lutze et al., 2013) or function-based (Freund \& Sundmacher, 2008) approaches hold great potential for exploring a vast search space for synthesis of these systems without hindering it with known unit operations. An excellent recent review and future outlook regarding process intensification is given in Baldea (2014).

\section{Concluding Remarks}

The diversity of future raw materials, whether it is biological or fossil-based (e.g., shale gas), and the growth of personalized medicine may require synthesis of flexible processes that can operate under a wide range of conditions. The lack of physicochemical data for these raw materials and possible products offers unique opportunities for integration of experimental and process synthesis activities, and for development of surrogate and multi-scale modelling approaches. The stochastic and/or robust optimization approaches are expected to play a significant role due to inherent uncertainties. The author believes that as process synthesis tools and approaches contribute to the development of these systems, they in turn will fuel the development of new tools and approaches for process synthesis.

\section{Acknowledgements}

The author would like to acknowledge her former and current graduate and undergraduate students for stimulating discussions that contributed significantly to this paper. Also, the financial support 
provided by NSF CAREER Award No 1055974 and Chevron Energy Technology Company is greatly acknowledged.

\section{References}

Agrawal, R. (2001). Separations: Perspective of a process developer/designer. AIChE Journal, 47, 967-971.

Baldea, M. (2014). Multum in Parvo: A Process Intensification Retrospective and Outlook. In J. D. S. Mario R. Eden \& P. T. Gavin (Eds.), Computer Aided Chemical Engineering (Vol. Volume 34, pp. 15-24): Elsevier.

Barnicki, S. D., \& Siirola, J. J. (2004). Process synthesis prospective. Computers and Chemical Engineering, 28, 441-446.

BASF. (2014). BASF Factbook 2014. In.

Bayer. (2014). Bayer 2014 Annual Report. In.

Biegler, L. T., Lang, Y.-d., \& Lin, W. (2014). Multi-scale optimization for process systems engineering. Computers \& Chemical Engineering, 60, 17-30.

Bommareddy, S., Chemmangattuvalappil, N. G., Solvason, C. C., \& Eden, M. R. (2010). Simultaneous solution of process and molecular design problems using an algebraic approach. Computers \& Chemical Engineering, 34, 1481-1486.

Caballero, J. A., \& Grossmann, I. E. (2013). Synthesis of complex thermally coupled distillation systems including divided wall columns. AIChE Journal, 59, 1139-1159.

Casciato, M. J., Kim, S., Lu, J. C., Hess, D. W., \& Grover, M. A. (2012). Optimization of a Carbon Dioxide-Assisted Nanoparticle Deposition Process Using Sequential Experimental Design 
with Adaptive Design Space. Industrial \& Engineering Chemistry Research, 51, 43634370.

Christian, B., \& Cremaschi, S. (2014). A Quick Knapsack Heuristic Solution for Pharmaceutical R\&D Pipeline Management Problems. Computer Aided Chemical Engineering (in press).

Colvin, M., \& Maravelias, C. T. (2008). A stochastic programming approach for clinical trial planning in new drug development. Computers \& Chemical Engineering, 32, 2626-2642.

Company, T. D. C. (2014). The Dow Chemical Company 2014 Annual Report. In.

Cremaschi, S. (2014). A perspective on process synthesis: Challenges and prospects. In J. D. S. Mario R. Eden \& P. T. Gavin (Eds.), Computer Aided Chemical Engineering (Vol. Volume 34, pp. 35-44): Elsevier.

Domínguez, L. F., \& Pistikopoulos, E. N. (2013). A quadratic approximation-based algorithm for the solution of multiparametric mixed-integer nonlinear programming problems. AIChE Journal, 59, 483-495.

Douglas, J. M. (1988). Conceptual Design of Chemical Processes. Singapore: Mc Gras-Hill Book Co. .

DuPont. (2013). Dupont 2013 Data Book. In.

Eason, J., \& Cremaschi, S. (2014). Adaptive sequential sampling for surrogate model generation with artificial neural networks. Computers \& Chemical Engineering, 68, 220-232.

Fahmi, I., \& Cremaschi, S. (2012). Process synthesis of biodiesel production plant using artificial neural networks as the surrogate models. Computers \& Chemical Engineering, 46, 105123. 
Fahmi, I., Nuchitprasittichai, A., \& Cremaschi, S. (2014). A new representation for modeling biomass to commodity chemicals development for chemical process industry. Computers \& Chemical Engineering, 61, 77-89.

Feinberg, M. (2002). Toward a Theory of Process Synthesis. Industrial \& Engineering Chemistry Research, 41, 3751-3761.

Felbab, N., Patel, B., El-Halwagi, M. M., Hildebrandt, D., \& Glasser, D. (2013). Vapor recompression for efficient distillation. 1. A new synthesis perspective on standard configurations. AIChE Journal, 59, 2977-2992.

Fernandes, F. A. N. (2006). Optimization of Fischer-Tropsch Synthesis Using Neural Networks. Chemical Engineering \& Technology, 29, 449-453.

Freund, H., \& Sundmacher, K. (2008). Towards a methodology for the systematic analysis and design of efficient chemical processes. Part 1. From unit operations to elementary process functions. Chemical Engineering and Processing: Process Intensification, 47, 2051-2060.

Furman, K. C., \& Sahinidis, N. V. (2004). Approximation algorithms for the minimum number of matches problem in heat exchanger network synthesis. Industrial and Engineering Chemistry Research, 43, 3554-3565.

Giridhar, A., \& Agrawal, R. (2010). Synthesis of distillation configurations. II: A search formulation for basic configurations. Computers \& Chemical Engineering, 34, 84-95.

Grossmann, I. E., Aguirre, P. A., \& Barttfeld, M. (2005). Optimal synthesis of complex distillation columns using rigorous models. Computers \& Chemical Engineering, 29, 1203-1215.

Grossmann, I. E., \& Guillén-Gosálbez, G. (2010). Scope for the application of mathematical programming techniques in the synthesis and planning of sustainable processes. Computers \& Chemical Engineering, 34, 1365-1376. 
Gupta, V., \& Grossmann, I. E. (2014). A new decomposition algorithm for multistage stochastic programs with endogenous uncertainties. Computers \& Chemical Engineering, 62, 62-79. Harmsen, G. J. (2007). Reactive distillation: The front-runner of industrial process intensification: A full review of commercial applications, research, scale-up, design and operation. Chemical Engineering and Processing: Process Intensification, 46, 774-780.

Harmsen, J. (2010). Process intensification in the petrochemicals industry: Drivers and hurdles for commercial implementation. Chemical Engineering and Processing: Process Intensification, 49, 70-73.

Held, H., \& Woodruff, D. (2005). Heuristics for Multi-Stage Interdiction of Stochastic Networks. Journal of Heuristics, 11, 483-500.

Henao, C. A., \& Maravelias, C. T. (2010). Surrogate-based superstructure optimization framework. AIChE Journal, n/a-n/a.

Henao, C. A., \& Maravelias, C. T. (2011). Surrogate-based superstructure optimization framework. AIChE Journal, 57, 1216-1232.

Hendry, J. E., Rudd, D. F., \& Seader, J. D. (1973). Synthesis in the design of chemical processes. AIChE Journal, 19, 1-15.

Jeżowski, J. (2010). Review of Water Network Design Methods with Literature Annotations. Industrial \& Engineering Chemistry Research, 49, 4475-4516.

Johannes, T. W., \& Zhao, H. (2006). Directed evolution of enzymes and biosynthetic pathways. Current Opinion in Microbiology, 9, 261-267.

Kiss, A. A. (2014). Distillation technology - still young and full of breakthrough opportunities. Journal of Chemical Technology \& Biotechnology, 89, 479-498. 
Lutze, P., Babi, D. K., Woodley, J. M., \& Gani, R. (2013). Phenomena based methodology for process synthesis incorporating process intensification. Industrial and Engineering Chemistry Research, 52, 7127-7144.

Lutze, P., Gani, R., \& Woodley, J. M. (2010). Process intensification: A perspective on process synthesis. Chemical Engineering and Processing: Process Intensification, 49, 547-558.

Malwade, C., Qu, H., Rong, B. G., \& Christensen, L. P. (2012). Conceptual process synthesis for isolation and purification of natural products from plants - A case study of artemisinin from Artemisia annua. In (Vol. 31, pp. 1707-1711).

Misener, R., \& Floudas, C. (2014). ANTIGONE: Algorithms for coNTinuous / Integer Global Optimization of Nonlinear Equations. Journal of Global Optimization, 1-24.

Morar, M., \& Agachi, P. S. (2010). Review: Important contributions in development and improvement of the heat integration techniques. Computers \& Chemical Engineering, 34, 1171-1179.

Motard, R. L. (1979). Process synthesis. Computers \& Chemical Engineering, 3, 13-15.

Nfor, B. K., Ahamed, T., van Dedem, G. W. K., van der Wielen, L. A. M., van de Sandt, E. J. A. X., Eppink, M. H. M., \& Ottens, M. (2008). Design strategies for integrated protein purification processes: Challenges, progress and outlook. Journal of Chemical Technology and Biotechnology, 83, 124-132.

Nfor, B. K., Ahamed, T., van Dedem, G. W. K., Verhaert, P. D. E. M., van der Wielen, L. A. M., Eppink, M. H. M., van de Sandt, E. J. A. X., \& Ottens, M. (2013). Model-based rational methodology for protein purification process synthesis. Chemical Engineering Science, 89, 185-195. 
Nuchitprasittichai, A., \& Cremaschi, S. (2011). Optimization of CO2 capture process with aqueous amines using response surface methodology. Computers \& Chemical Engineering, 35, 1521-1531.

Nuchitprasittichai, A., \& Cremaschi, S. (2013). Optimization of CO2 Capture Process with Aqueous Amines-A Comparison of Two Simulation-Optimization Approaches. Industrial \& Engineering Chemistry Research, 52, 10236-10243.

Polykarpou, E. M., Dalby, P. A., \& Papageorgiou, L. G. (2012). A novel efficient optimisation system for purification process synthesis. Biochemical Engineering Journal, 67, 186-193.

Qian, Z., Seepersad, C. C., Joseph, V. R., Allen, J. K., \& Wu, C. F. J. (2006). Building Surrogate Models Based on Detailed and Approximate Simulations. Journal of Mechanical Design, $128,668-677$.

Shah, V. H., \& Agrawal, R. (2010). A matrix method for multicomponent distillation sequences. AIChE Journal, 56, 1759-1775.

Shenvi, A. A., Shah, V. H., \& Agrawal, R. (2013). New multicomponent distillation configurations with simultaneous heat and mass integration. AIChE Journal, 59, 272-282.

Siirola, J. J., \& Rudd, D. F. (1971). Computer-Aided Synthesis of Chemical Process Designs. From Reaction Path Data to the Process Task Network. Industrial \& Engineering Chemistry Fundamentals, 10, 353-362.

Skiborowski, M., Harwardt, A., \& Marquardt, W. (2013). Conceptual design of distillation-based hybrid separation processes. Annual Review of Chemical and Biomolecular Engineering, 4, 45-68.

Smith, J. D., Neto, A. A., Cremaschi, S., \& Crunkleton, D. W. (2012). CFD-Based Optimization of a Flooded Bed Algae Bioreactor. Industrial \& Engineering Chemistry Research. 
Stankiewicz, A. I., \& Moulijn, J. A. (2000). Process Intensification: Transforming Chemical Engineering. Chemical Engineering Progress, January, 22 - 34.

Tawarmalani, M., \& Sahinidis, N. V. (2005). A polyhedral branch-and-cut approach to global optimization. Mathematical Programming, 103, 225-249.

Urselmann, M., Barkmann, S., Sand, G., \& Engell, S. (2011). Optimization-based design of reactive distillation columns using a memetic algorithm. Computers \& Chemical Engineering, 35, 787-805.

Van Gerven, T., \& Stankiewicz, A. (2009). Structure, Energy, Synergy, Time-The Fundamentals of Process Intensification. Industrial \& Engineering Chemistry Research, 48, 2465-2474.

Viswanath, K., Peeta, S., \& Salman, F. S. (2004). Investing in the links of a stochastic network to minimize expected shorthest path lengths. Institute for Research in the Behavioral, Economics, and Management Sciences, Paper No. 1167.

Viswanathan, J., \& Grossmann, I. E. (1990). A combined penalty function and outerapproximation method for MINLP optimization. Computers \& Chemical Engineering, 14, 769-782.

Westerberg, A. W. (2004). A retrospective on design and process synthesis. Computers and Chemical Engineering, 28, 447-458.

Yuan, Z., \& Chen, B. (2012). Process synthesis for addressing the sustainable energy systems and environmental issues. AIChE Journal, 58, 3370-3389.

Yuan, Z., Chen, B., \& Gani, R. (2013). Applications of process synthesis: Moving from conventional chemical processes towards biorefinery processes. Computers \& Chemical Engineering, 49, 217-229. 


\section{List of Figures}

Figure 1. A graphical representation of process synthesis

Figure 2. Number of process synthesis publications (1972 - 2013) - Reactor networks: search in All Fields for the exact phrase "reactor network synthesis", distillation: search in All Fields for either of the following exact phrases "distillation column synthesis", "separation network synthesis", "distillation sequence synthesis", "distillation synthesis", "separation train synthesis", heat-exchanger networks: search in All Fields for the exact phrase "heat exchanger network synthesis", and overall flowsheeting: search in All Fields for either of the following exact phrases "flowsheet synthesis", “complete flowsheet synthesis", "general flowsheet synthesis".

Figure 3. Basic distillation column configurations for separation of a tertiary mixture.

Figure 4. Example unconventional distillation columns, (a) vapor recompression distillation, (b) heat integrated distillation, (c) membrane distillation, (d) dividing wall columns, and (e) reactive distillation

Figure 5. Example hybrid separation sequences (a) liquid-liquid extraction and distillation, and (b) membrane-assisted distillation 


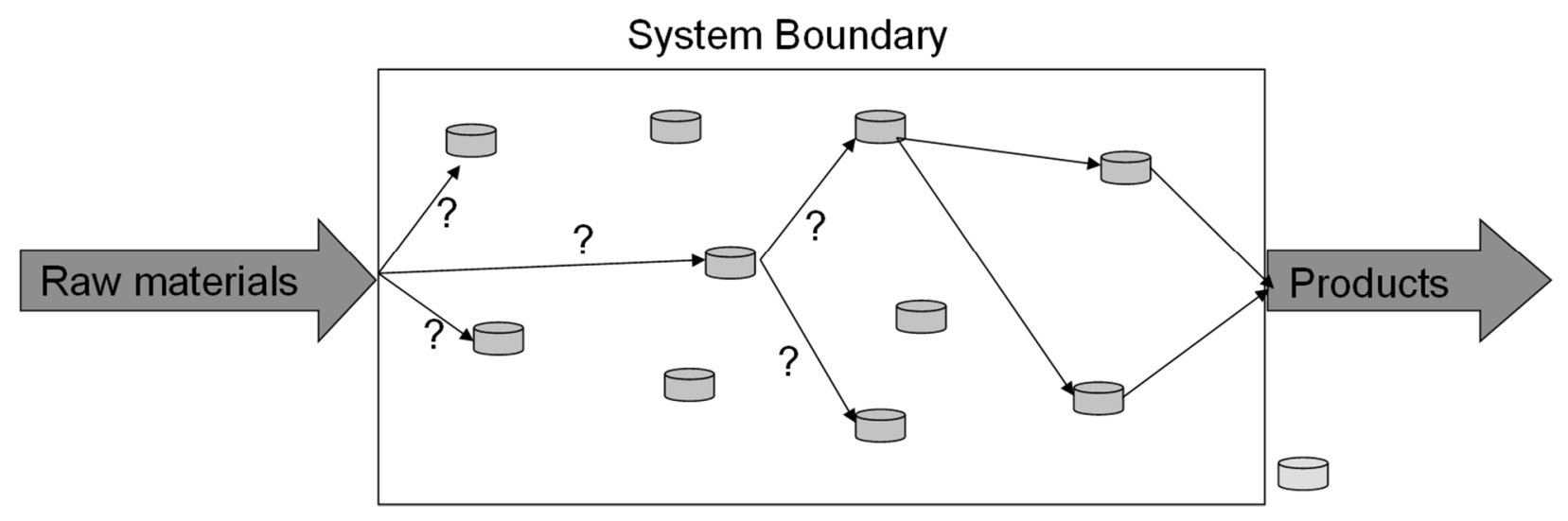

Figure 1. A graphical representation of process synthesis 


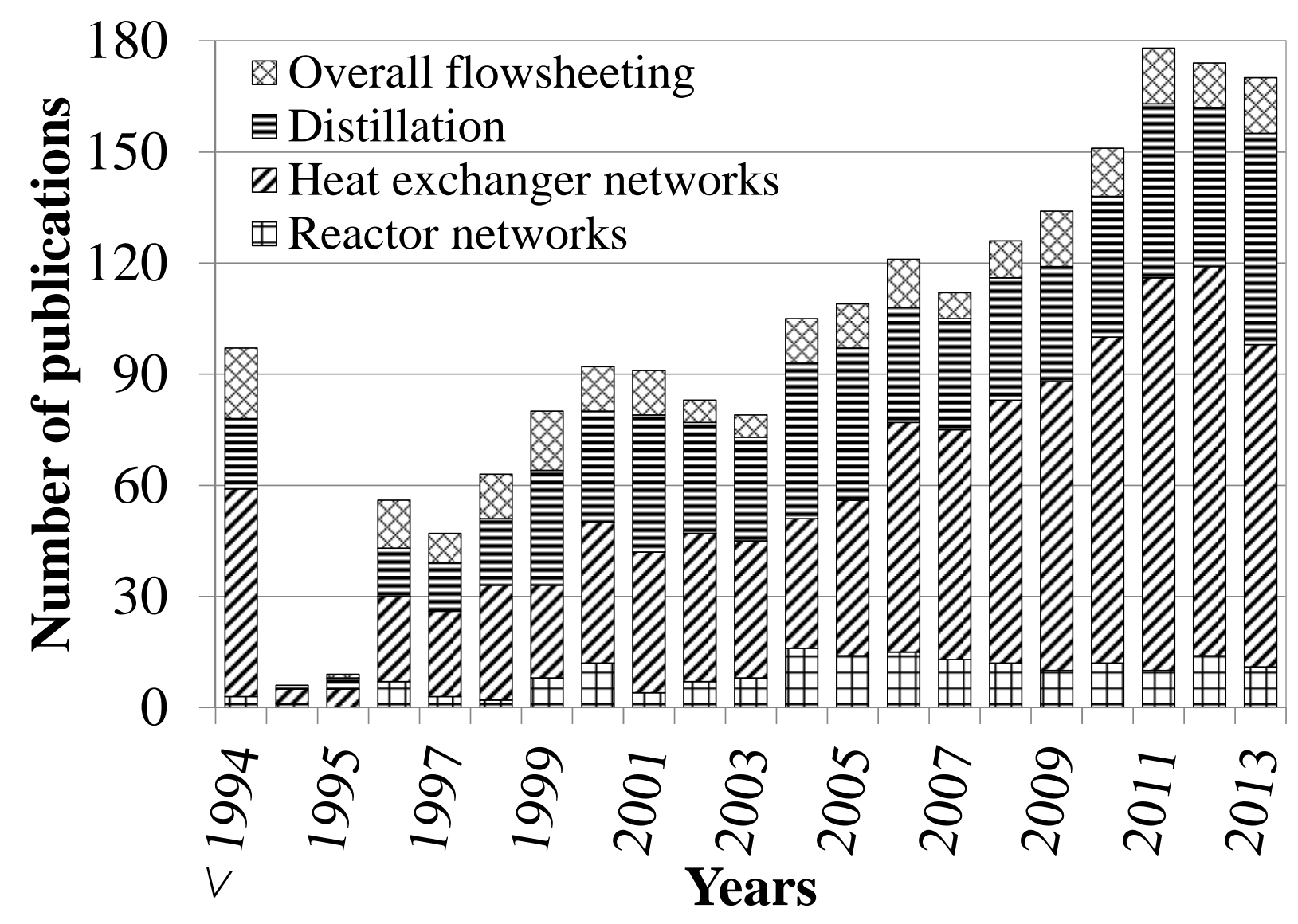

Figure 2. Number of process synthesis publications (1972 - 2013) - Reactor networks: search in All Fields for the exact phrase "reactor network synthesis", distillation: search in All Fields for either of the following exact phrases "distillation column synthesis", "separation network synthesis”, “distillation sequence synthesis”, “distillation synthesis”, "separation train synthesis”, heat-exchanger networks: search in All Fields for the exact phrase "heat exchanger network synthesis”, and overall flowsheeting: search in All Fields for either of the following exact phrases "flowsheet synthesis”, “complete flowsheet synthesis”, "general flowsheet synthesis”. 

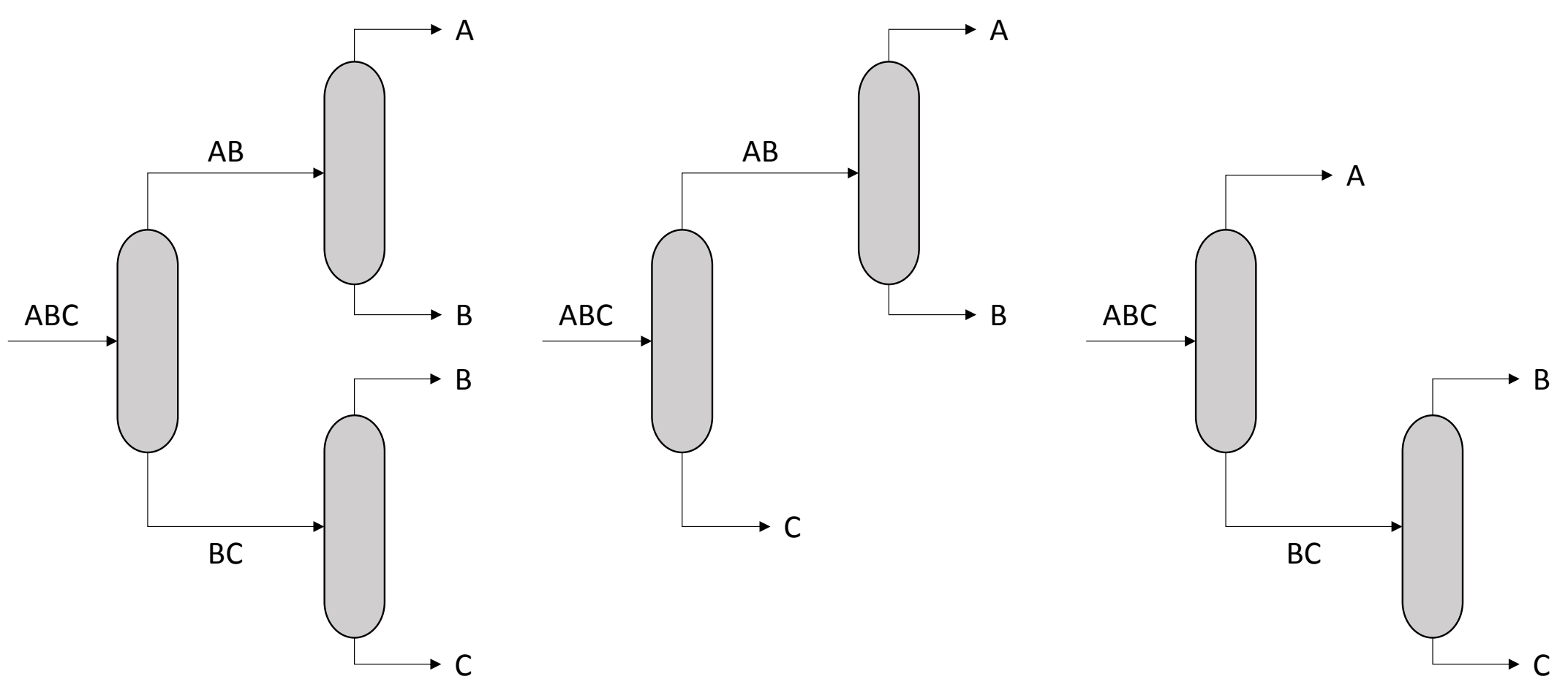

Figure 3. Basic distillation column configurations for separation of a tertiary mixture. 


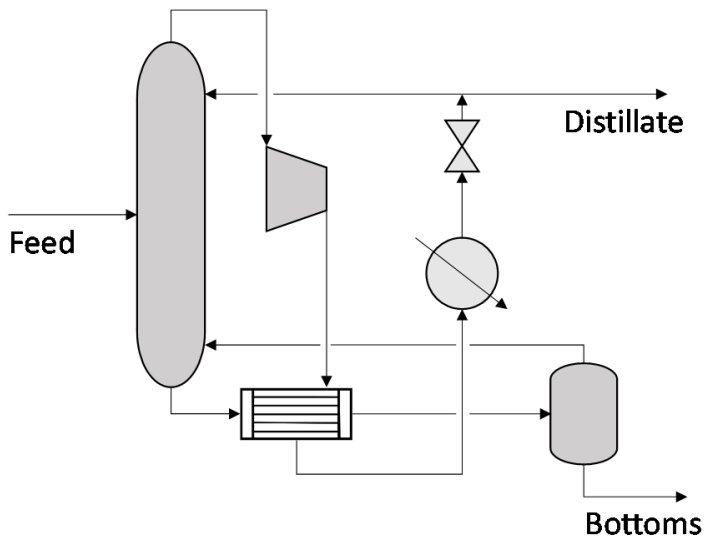

(a)

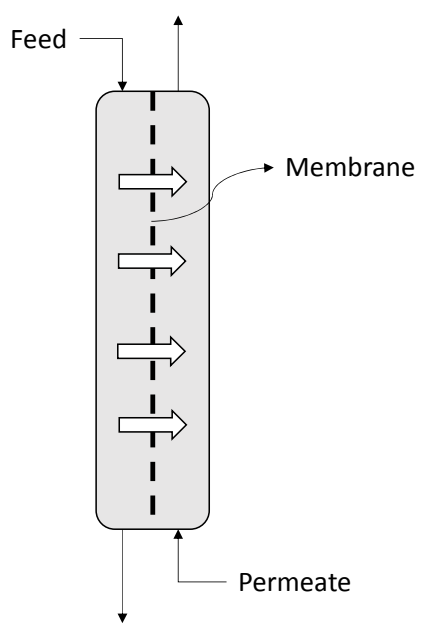

(c)

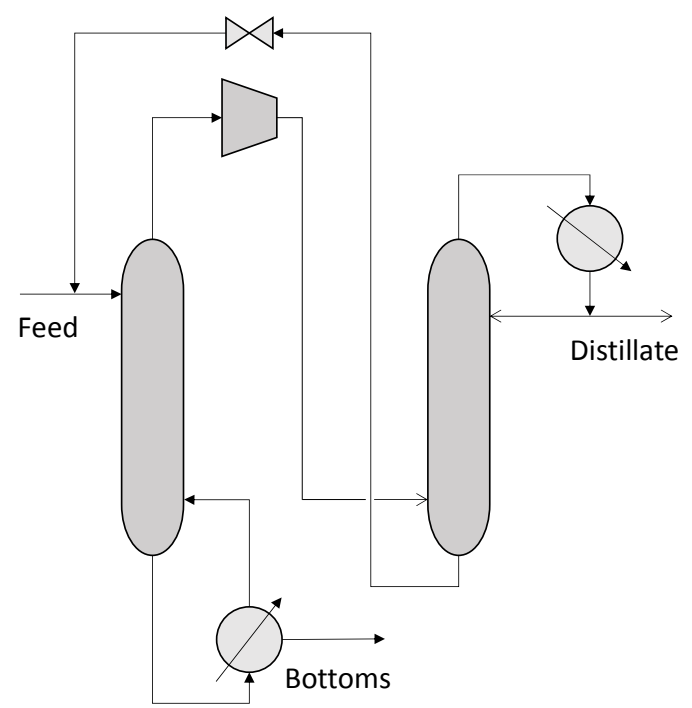

(b)

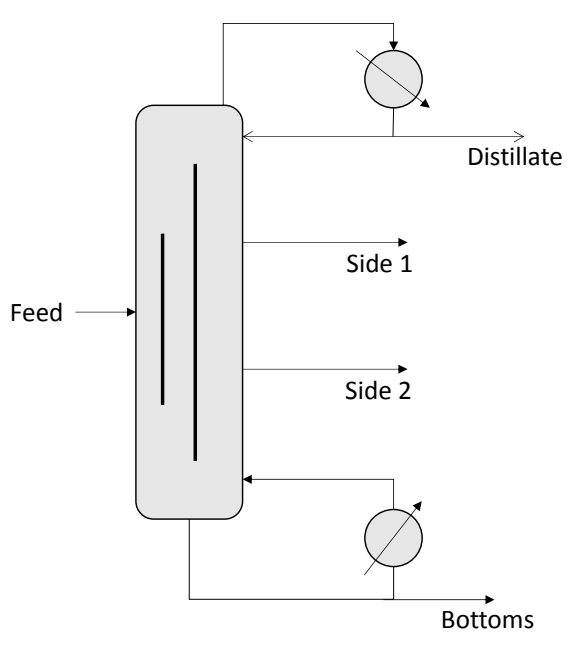

(d)

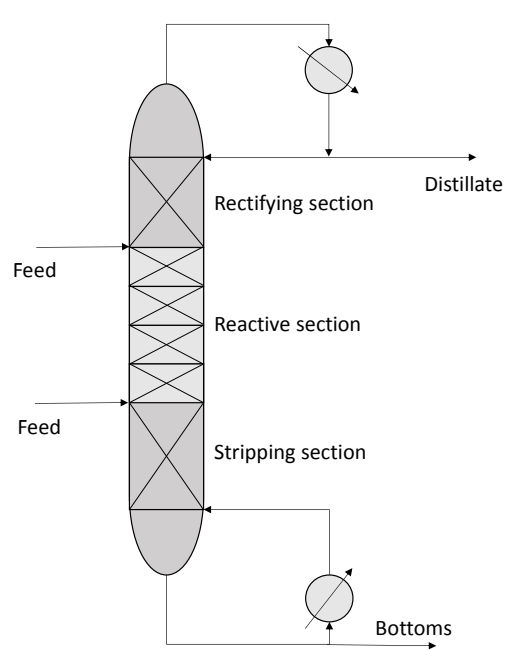

(e)

Figure 4. Example unconventional distillation columns, (a) vapor recompression distillation, (b) heat integrated distillation, (c) membrane distillation, (d) dividing wall columns, and (e) reactive distillation 


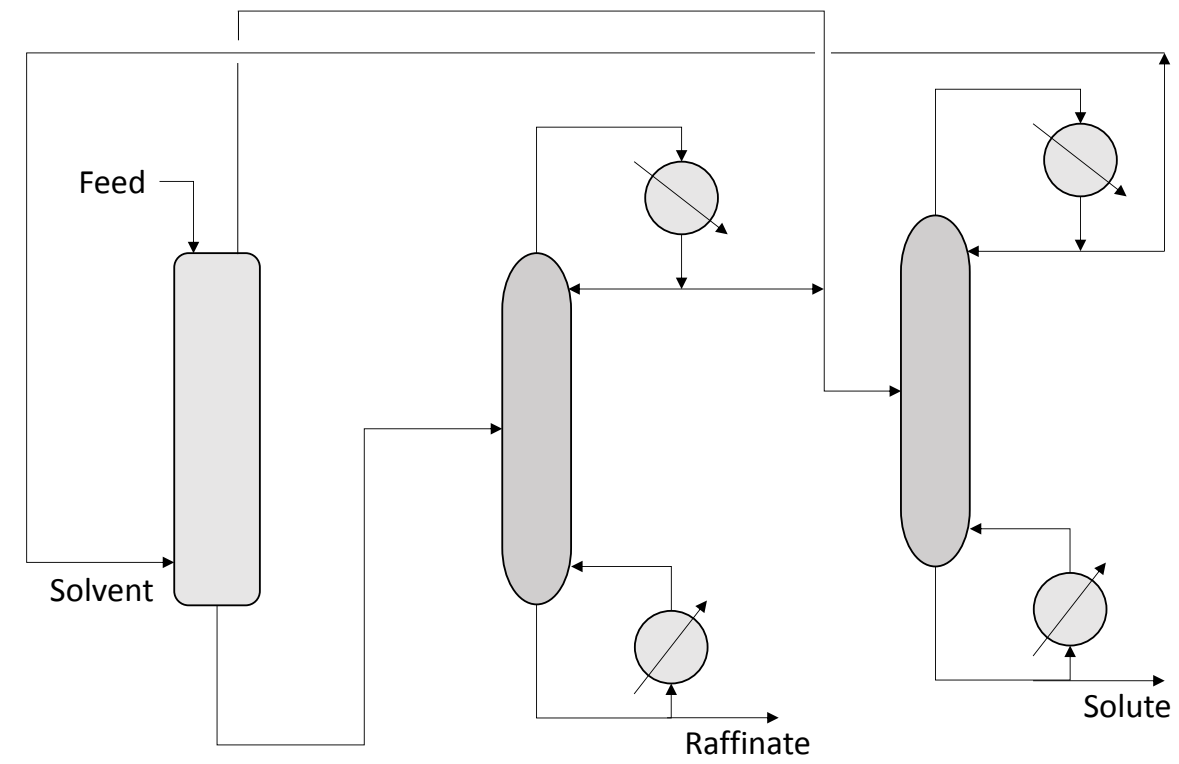

(a)

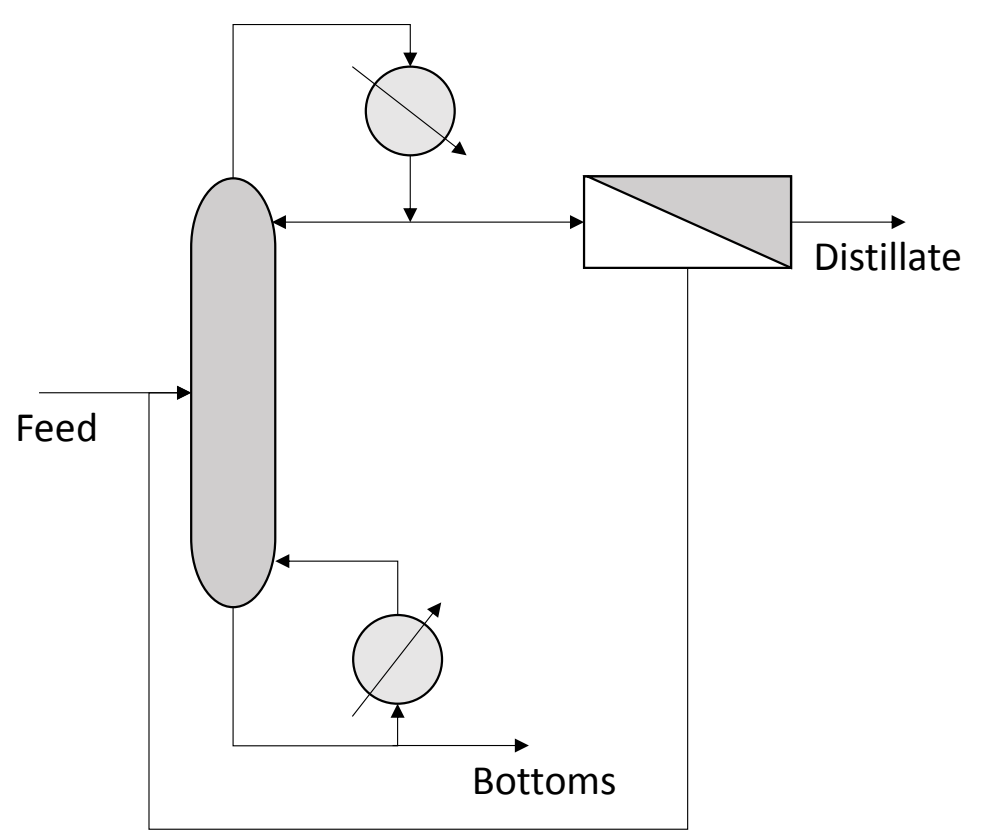

(b)

Figure 5. Example hybrid separation sequences (a) liquid-liquid extraction and distillation, and (b) membrane-assisted distillation 